\title{
Second-to-fourth digit ratio and seborrheic dermatitis in males: a cross-sectional study ${ }^{*}$
}

\author{
Zeynep Gizem Kaya İslamoğlu ${ }^{1}$
}

DOI: http:/ / dx.doi.org/10.1590/abd1806-4841.20198578

\begin{abstract}
BACKGROUND: Seborrheic dermatitis is a common disease characterized by the erythematous plaques with oily-yellow desquamation. Increased sebaceous gland activity by androgenic hormones has played a role in the etiology of the disease. The second-to-fourth digit (2D:4D) ratio is thought to be a marker of prenatal androgen exposure.

Овјестіves: To investigate the association between 2D:4D ratios and seborrheic dermatitis in a male population.

METHODS: Healthy male controls and patients with seborrheic dermatitis were included in this study. One hundred seborrheic dermatitis patients and 120 healthy controls, aged 17-59, were enrolled. A digital Vernier caliper was used to measure the finger lengths. Seborrheic dermatitis severity was assessed using the Seborrheic Dermatitis Area and Severity Index (SDASI). RESULTS: The 2D:4D ratios of the patients $(\bar{x}=0.977)$ were significantly lower than those of the controls $(\bar{x}=1.050)$ for right hands $(t=6.948 ; p=0.000 ;>0.05)$. No similar relationship was found between the 2D:4D ratio for left hands $(t=0.901 ; p=0.368 ;>0.05)$. Seborrheic dermatitis severity was negatively correlated with 2D:4D ratios of right hands $(r=-0.391 ; p=0.000-0.05)$.

STUDY LIMITATIONS: One of the main limitations of this study was the small sample, which got a head of us from acquiring certain findings about the 2D:4D ratio and seborrheic dermatitis. The other limitation is that the patient selection did not reflect the general population, as a single clinic was studied.

CONCLUSION: To the authors' knowledge, this is the first study examining the relationship between 2D:4D ratios and seborrheic dermatitis. The result of this study may indicate a line of investigation and can support the theory of prenatal androgen exposure.
\end{abstract}

Keywords: Androgens; Dermatitis, seborrheic; Fingers; Hyperandrogenism

\section{INTRODUCTION}

Seborrheic dermatitis (SD) is a chronic relapsing inflammatory skin disorder clinically characterized by scaling and poorly defined erythematous patches. ${ }^{1}$ It has a distinctive distribution in areas rich with sebaceous glands, such as the scalp, eyebrows, glabella, nasolabial and nasofacial folds, cheeks, periauricular skin, and presternal and interscapular areas. ${ }^{2}$ SD especially affects adolescence and post-adolescence, and is seen in both the general and young population, with ratios of $3 \%-5 \%$ and $1 \%-3 \%$, respectively. ${ }^{3,4}$ It is most common in men, starting in puberty and peaking in the forties. ${ }^{2} \mathrm{SD}$ is seen at higher rates in immunocompromised patients, and can be divided into infantile-onset SD and adult-onset SD. ${ }^{5}$ It is a multifactorial disease with many factors that play a role in etiology, although the exact cause of SD is unknown. The factors include stress, androgens, depression, fatigue, vascular disorders (which can be microangiopathic), atmospheric changes such as pressure alteration, seasonal changes, clothing, eating habits and food allergies, autoimmunity, climacteric changes, Malassezia furfur, taking medications for different reasons, and riboflavin, pyridoxine, or biotin

Received 27 June 2018.

Accepted 30 August 2018

* Study performed at the Department of Dermatology, School of Medicine, Selcuk University, Konya, Turkey.

Financial support: None.

Conflict of Interest: None

Department of Dermatology, School of Medicine, Selcuk University, Konya, Turkey.

MAILING AdDRESS:

Zeynep Gizem Kaya İslamoğlu

E-mail: gizemislamoglu@hotmail.com 
deficiency. ${ }^{6}$ Sebaceous glands in the skin are targeted by androgens. Androgenic hormones have an important role in the etiology of the disease, which is known to be increase sebaceous gland activity. ${ }^{7}$

The digit ratio 2D:4D refers to the ratio of the length of the second finger to that of the fourth finger. ${ }^{8}$ Some evidence suggests that it is an indicator of prenatal exposure to sex hormones and sensitivity to androgens. ${ }^{8-10}$ It is found that the second-to-fourth digit (2D:4D) ratio is smaller in males when compared with females. ${ }^{11}$ Exposure to higher androgen levels in the prenatal period being connected to a lower 2D:4D ratio in both sexes is also suggested in some studies. ${ }^{12,13}$ Furthermore, a low 2D:4D ratio has also been detected in female patients diagnosed with congenital adrenal hyperplasia. ${ }^{14}$

There are many studies that have suggested the association of the 2D:4D ratio and diseases such as acne vulgaris; androgenetic alopecia; hormone levels, sperm count, muscle mass; myocardial infarction; psychiatric disorders such as autism; eating and language disorders; and some cancers. ${ }^{8,9,11-19}$ However, there are no articles on seborrheic dermatitis and digit ratio. The role of androgens in seborrheic dermatitis is well known. In this study, the authors aimed to evaluate the relationship between the 2D:4D ratios and seborrheic dermatitis and the severity of the disease in males.

\section{METHODS}

The study was initiated after approval by the research and ethics committee, №. 2017/142. Informed consent was obtained from all participants. The study group included patients with SD and healthy controls who hadn't been diagnosed with SD during their life. The groups were chosen from patients and healthy subjects who attended this dermatology clinic and the local community between May 2017 and December 2017. A form was used to register the demographic data and disease information. SD severity was measured by using the Seborrheic Dermatitis Area and Severity Index (SDASI). The SDASI is a new tool for scoring the disease severity in daily clinical practice. Disease severity, erythema, dandruff, and pruritus were evaluated and scored between 1 and 3 points. According to the scoring, (1) corresponded with mild, (2) corresponded with moderate, and (3) corresponded with severe. The score of each site was multiplied by the constant for the area (forehead $=0.1$, scalp $=0.4$, nasolabial $=0.1$, eyebrow $=0.1$, postauricular $=0.1$, auricular $=0.1$, intermammary $=0.2$, back $=0.2$, and cheek or chin $=0.1$ ) and the sum was determined as the SDASI score. Subjective assessment of pruritus was scored by the patients as follows: $0=$ none, $1=$ mild, $2=$ moderate, and $3=$ severe .

The lengths of the second-to-fourth digit were measured using a digital Vernier caliper with a resolution of $0.01 \mathrm{~mm}$. The same person performed the measurements and measurements were taken directly from the fingers rather than from indirect methods to eliminate measurement differences. Measurements of the fingers were made on the palmar surface of the hand from the metacarpophalangeal crease to the tip and were taken from both the right and left palmar areas. The 2D:4D ratio was obtained by dividing the length of the second finger by the length of the fourth finger.

The data obtained in the study were analyzed using the SPSS for Windows v. 22.0 program. Number, percentage, mean, and standard deviation were used as the descriptive statistical methods in the evaluation of the data. Student's $t$-test was used to compare quantitative continuous data between two independent groups. Cohen's effect size was calculated to quantify the extent of the difference in 2D:4D between individuals with seborrheic dermatitis patients and controls. Spearman's rank correlation was used to compare quantitative continuous data among more than two independent groups (2D:4D and SDASI; 2D:4D and the pruritus intensity). The findings were evaluated with a $95 \%$ confidence interval and at the $5 \%$ significance level. Statistical significance was assigned at $\mathrm{p}<0.05$

\section{RESULTS}

A total of 100 men patients and 120 healthy controls were enrolled in the study. The mean age of the SD group was $27.44 \pm$ 9.266, the mean age of the healthy controls was $27.292 \pm 9.448$. The mean age did not differ significantly between the groups $(t=0.117$; $p=0.907 ;>0.05)$. The distribution of SDASI and pruritus is shown in table 1 . The left hand 2D:4D ratios did not differ significantly from the healthy control group $(t=0.901 ; p=0.368 ;>0.05)$. However, the right hand 2D:4D scores $(\bar{x}=1.050)$ of the control group were higher than the right hand 2D:4D scores $(\bar{x}=0.977)$ of the patient group $(t=6.948 ; p=0.000 ;>0.05)$. Thus, it was found that the right hand 2D:4D of the SD group was significantly lower than that of healthy controls (Table 2). No significant relationship was found between SDASI scores and left hand 2D:4D ratios ( $p>0.05)$. However, there was a relation between right hand 2D:4D ratios and SDASI scores $(p<0.050)$. As a result of Spearman's rank correlation analysis to determine the sources of differences, the right hand 2D:4D ratios were higher in mild SDASI scores than in moderate and severe scores (Table 3). Pruritus scores were not significantly different in the left and right hand 2D:4D ratios (Table 3).

\section{DISCUSSION}

To the best of the authors' knowledge, this is the first study to examine the relationship between 2D:4D ratio and seborrheic dermatitis in males. This study found a lower 2D:4D ratio in the right hand, but no similar relationship was detected in the left hand. In addition, this study found a relationship between disease severity

\begin{tabular}{|c|c|c|c|}
\hline & & Frequency (n) & Percent (\%) \\
\hline \multirow[t]{4}{*}{ SDASI scores } & Mild & 44 & 44.0 \\
\hline & Moderate & 48 & 48.0 \\
\hline & Severe & 8 & 8.0 \\
\hline & Total & 100 & 100.0 \\
\hline \multirow[t]{5}{*}{ Pruritus } & None & 16 & 16.0 \\
\hline & Mild & 42 & 42.0 \\
\hline & Moderate & 38 & 38.0 \\
\hline & Severe & 4 & 4.0 \\
\hline & Total of patients & 100 & 100.0 \\
\hline
\end{tabular}

SD, seborrheic dermatitis; SDASI, Seborrheic Dermatitis Area and Severity Index 


\begin{tabular}{|c|c|c|c|c|c|c|c|}
\hline & \multicolumn{2}{|c|}{ Patients $(n=100)$} & \multicolumn{2}{|c|}{ Controls $(n=120)$} & \multirow[b]{2}{*}{$\mathbf{t}$} & \multirow[b]{2}{*}{ p } & \multirow[b]{2}{*}{ Cohen's c } \\
\hline & Ort & Ss & Ort & Ss & & & \\
\hline Age & 27.440 & 9.266 & 27.292 & 9.448 & & & \\
\hline Left hand 2D & 68.474 & 6.074 & 68.128 & 5.586 & 0.439 & 0.661 & 0.059 \\
\hline Left hand $4 \mathrm{D}$ & 71.842 & 5.473 & 72.151 & 5.187 & -0.429 & 0.668 & -0.058 \\
\hline Left hand 2D:4D & 0.956 & 0.081 & 0.946 & 0.072 & 0.901 & 0.368 & 0.131 \\
\hline Right hand 2D & 70.858 & 6.990 & 72.472 & 5.012 & -1.989 & 0.055 & -0.265 \\
\hline Right hand 4D & 72.746 & 5.057 & 69.158 & 5.403 & 5.048 & 0.000 & 0.686 \\
\hline Right hand 2D:4D & 0.977 & 0.100 & 1.050 & 0.053 & -6.948 & 0.000 & -0.912 \\
\hline
\end{tabular}

SD, seborrheic dermatitis; 2D:4D, ratio of the second digit to the fourth digit.

\begin{tabular}{llllll}
\multicolumn{5}{c}{ TABLE 3: Correlations between the severity of SD and intensity } \\
of pruritus and 2D:4D ratios
\end{tabular}

$*<0.05 ; \mathrm{b}<0.01$.

SD, seborrheic dermatitis; SDASI, Seborrheic Dermatitis Area and Severity Index; 2D:4D, ratio of the second digit to the fourth digit.

and 2D:4D ratio on the right hand. As the disease severity increased, the $2 \mathrm{D}: 4 \mathrm{D}$ ratio decreased.

In previous studies, it has been suggested that there is a relationship between lower 2D:4D ratios and prenatal androgen exposure. ${ }^{9,12,20,21}$ Seborrheic dermatitis is a common relapsing inflammatory skin disease with unclear pathophysiological mechanisms. ${ }^{5}$ Several hypotheses are assumed to play an etiological role in the formation of seborrheic dermatitis; the most important factor is the increase of sebaceous gland secretions. The sebaceous glands are the target organs of androgens in the skin; the glands grow in the presence of androgens and release more sebaceous secretions. ${ }^{7}$ In a study, it was found that men were twice as likely to develop seborrheic dermatitis than women. They explained this association due to hormonal differences between men and women. ${ }^{22}$ Again, after applied adrenalectomy or antiandrogen therapy, patients have reduced fat secretion. ${ }^{7}$ With these studies, the effect of androgens on seborrheic dermatitis can be better understood.

Recent studies have shown an association between digit ratio and some dermatological diseases. In a study of androgenetic alopecia (AGA), Bilgiç et al. found significantly lower 2D:4D ratios for the right hand in the AGA group than in healthy controls; however, no significant difference for the left hand were found. In addition, they didn't find a significant correlation between AGA severity and 2D:4D digit ratio. ${ }^{23}$ In another study of AGA, Unal found a significant lower digit ratio $2 \mathrm{D}: 4 \mathrm{D}$ for the left hand in the AGA group, but no difference was for the right hand $v s$. healthy controls and no correlation was determined between the 2D:4D ratio and AGA severity. ${ }^{16}$ A study on acne showed that that a lower 2D:4D ratio is related to acne vulgaris in females, but not related in males. ${ }^{24}$ In their next report, they examined the relationship between the 2D:4D ratio and skin sebum levels in females with acne vulgaris. They found that lower 2D:4D ratios correlate with higher rates of sebum secretion in acne patients. ${ }^{15}$ Another study assessed the correlation between the 2D:4D ratio and acne, and acne-related disease variables and hormone levels in individuals with acne. They did not find a significant difference between acne patients and the control group independent of sex..$^{25}$ Therefore they suggested that prenatal androgen exposure may induce the development of some related diseases, together with other etiological factors.

Fetal sex hormones and genetic factors may influence the establishment of the 2D:4D ratios. It has been discussed that the right hand digit ratio may be a better indicator of prenatal androgenization than the left hand ratio. ${ }^{12,26}$ However, another study suggested that the genetic contribution to variations in the 2D:4D ratio tended to be stronger in the left hand..$^{27}$ The present study showed a correlation with the right hand digit ratio, similar to the first two studies.

Regarding a possible hypothesis, the authors expected that lower 2D:4D ratios would show a negative relation with SD severity. This correlation was found at the end of the study. Again, it was expected that lower 2D:4D ratios would be found in the right hand. This is because characteristics that differ between the sexes show a tendency for the male form of the feature to be most strongly 
expressed on the right side of the body. This may mean that right 2D:4D is more sensitive to the effects of testosterone. ${ }^{28}$ The difference between 2D:4D ratios on the right and left sides can thus be explained. We had already defined all of these hypotheses at the end.

In summary, this study detected the relationship between 2D:4D ratios and seborrheic dermatitis in men. Lower right hand digit ratios were found, as well as a correlation with disease severity. At the end of the study, it was determined that the 2D:4D ratio can be a good predictor of the development of seborrheic dermatitis, because the digit ratio does not change after birth. Because the relationship between digit ratio and seborrheic dermatitis is a new subject, the authors believe that further studies with large case and control groups will support this hypothesis clearly.

\section{CONCLUSIONS}

In conclusion, this study found that lower right hand 2D:4D digit ratios can be a predictor of seborrheic dermatitis development in males. In addition, this ratio is correlated to disease severity. Further studies are required for verification. $\square$

\section{ACKNOWLEDGMENTS}

What's new: The association between the 2D:4D ratio and seborrheic dermatitis may result from the effects of the prenatal endocrine environment on the sebaceous glands.

\section{REFERENCES}

1. Bukvić Mokos Z, Kralj M, Basta-Juzbašić A, Lakoš Jukić I. Seborrheic dermatitis: an update. Acta Dermatovenerol Croat. 2012;20:98-104.

2. Ooi ET, Tidman MJ. Improving the management of seborrhoeic dermatitis. Practitioner. 2014;258:23-6, 3.

3. Saçar T, Saçar H. Seborrheic dermatitis. J Clin Anal Med. 2010;2:57-60

4. Crespo Erchiga V, Ojeda Martos A, Vera Casaño A, Crespo Erchiga A, Sanchez Fajardo F. Malassezia globosa as the causative agent of pityriasis versicolor. $\mathrm{Br} \mathrm{J}$ Dermatol. 2000;143:799-803.

5. Gupta AK, Bluhm R. Seborrheic dermatitis. J Eur Acad Dermatol Venereol. 2004;18:13-26

6. Aksoy M, Arıca DA. Seborrheic dermatitis: review. Turkiye Klinikleri J Dermatol. 2016;26:90-100.

7. Sperling LC, Heimer WL 2nd. Androgen biology as a basis for the diagnosis and treatment of androgenic disorders in women II. J Am Acad Dermatol. 1993;28:901-16

8. Unal M, Urun Unal G, Balevi S, Tol H, Uyar M. The second to fourth digit ratio in acne vulgaris. Pediatr Dermatol. 2015;32:651-5

9. Manning JT, Wood S, Vang E, Walton J, Bundred PE, van Heyningen C, et al Second to fourth digit ratio (2D:4D) and testosterone in men. Asian J Androl. 2004;6:211-5.

10. Rivas MP, Moreira LM, Santo LD, Marques AC, El-Hani CN, Toralles MB. New studies of second and fourth digit ratio as a morphogenetic trait in subjects with congenital adrenal hyperplasia. Am J Hum Biol. 2014;26:559-61.

11. Manning JT, Barley L, Walton J, Lewis-Jones DI, Trivers RL, Singh D, et al. The 2nd:4th digit ratio, sexual dimorphism, population differences, and reproductive success: evidence for sexually antagonistic genes?. Evol Hum Behav. 2000;21:163-83

12. Manning J, Kilduff L, Cook C, Crewther B, Fink B. Digit ratio (2D:4D): a biomarker for prenatal sex steroids and adult sex steroids in challenge situations. Fron Endocrinol (Lausanne). 2014;5:9

13. Manning JT, Scutt D, Wilson J, Lewis-Jones DI. The ratio of 2nd to 4th digit length: a predictor of sperm numbers and concentrations of testosterone, luteinizing hormone and oestrogen. Hum Reprod. 1998;13:3000-4.

14. Okten A, Kalyoncu M, Yaris N. The ratio of second- and fourth digit lengths and congenital adrenal hyperplasia due to 21-hydroxylase deficiency. Early Hum Dev. 2002;70:47-54.
15. Bilgic Ö, Altınyazar $\mathrm{C}, \mathrm{H}$ ra $\mathrm{H}$, Doğdu $\mathrm{M}$. Investigation of the association of the second-to-fourth digit ratio with skin sebum levels in females with acne vulgaris Am J Clin Dermatol. 2015;16:559-64.

16. Unal M. Digit ratio $2 \mathrm{D}: 4 \mathrm{D}$ is a possible indicator for androgenetic alopecia in males. J Cosmet Dermatol. 2018;17:545-548.

17. Bunevicius $A$. The association of digit ratio (2d: $4 \mathrm{~d})$ with cancer: a systematic review and meta-analysis. Dis Markers. 2018;2018:7698193.

18. Font-Jordà A, Gamundí A, Nicolau Llobera MC, Aguilar-Mediavilla E. Use of the 2D:4D digit ratio as a biological marker of specific language disorders. An Pediatr (Barc). 2018 pii: S1695-4033(18)30105

19. Kyriakidis I, Papaioannidou P, Pantelidou V, Kalles V, Gemitzis K. Digit ratios and relation to myocardial infarction in Greek men and women. Gend Med. 2010;7:628-36.

20. Breedlove SM. Minireview: organizational hypothesis: instances of the fingerpost Endocrinology. 2010;151:4116-22

21. 21. Berenbaum SA, Bryk KK, Nowak N, Quigley CA, Moffat S. Fingers as a marke of prenatal androgen exposure. Endocrinology. 2009;150:5119-24.

22. Sanders MGH, Pardo LM, Franco OH, Ginger RS, Nijsten T. Prevalence and determinants of seborrhoeic dermatitis in a middle-aged and elderly population: the Rotterdam Study. Br J Dermatol. 2018;178:148-53.

23. Bilgic Ö, Altınyazar HC, Eryılmaz D, Tuğrul ZA. Are 2D:4D finger length ratios an indicator of androgenetic alopecia in males?. An Bras Dermatol. 2016;91:156-9.

24. Bilgiç 0 , Doğdu M, Islamoğlu GK, Altınyazar C. The relationship between the second to fourth digit ratio and acne vulgaris. J Eur Acad Dermatol Venereol. 2014:28:1340-3.

25. Unal M, Urun Unal G, Balevi S, Tol H, Uyar M. The second to fourth digit ratio in acne vulgaris. Pediatr Dermatol. 2015;32:651-5.

26. Hönekopp J, Watson S. Meta-analysis of digit ratio 2D:4D shows greater sex difference in the right hand. Am J Hum Biol. 2010;22:619-30.

27. Gobrogge KL, Breedlove SM, Klump KL. Genetic and environmental influences on 2D:4D finger length ratios: a study of monozygotic and dizygotic male and female twins. Arch Sex Behav. 2008:37:112-8.

28. Lutchmaya S, Baron-Cohen S, Raggatt P, Knickmeyer R, Manning JT. 2nd to 4th digit ratios, fetal testosterone and estradiol. Early Hum Dev. 2004;77:23-8. 